\title{
Laval théologique et philosophique
}

Bertrand Dumas, Dennis GIRA, dir., Le désir en question. Regards bouddhistes et chrétiens. Actes du colloque interreligieux du 5 au 8 juillet 2012 au centre théologique de Meylan-Grenoble. Lyon, PROFAC (coll. « PROFAC-Théo », 124), 2015, 186 p.

\section{André Couture}

Volume 72, numéro 1, février 2016

URI : https://id.erudit.org/iderudit/1038545ar

DOI : https://doi.org/10.7202/1038545ar

Aller au sommaire du numéro

Éditeur(s)

Faculté de philosophie, Université Laval

Faculté de théologie et de sciences religieuses, Université Laval

ISSN

0023-9054 (imprimé)

1703-8804 (numérique)

Découvrir la revue

Citer ce compte rendu

Couture, A. (2016). Compte rendu de [Bertrand DumAs, Dennis GIRA, dir., Le désir en question. Regards bouddhistes et chrétiens. Actes du colloque interreligieux du 5 au 8 juillet 2012 au centre théologique de Meylan-Grenoble. Lyon, PROFAC (coll. « PROFAC-Théo », 124), 2015, 186 p.] Laval théologique et philosophique, 72(1), 179-180. https://doi.org/10.7202/1038545ar

Tous droits réservés @ Laval théologique et philosophique, Université Laval, 2016
Ce document est protégé par la loi sur le droit d'auteur. L'utilisation des services d'Érudit (y compris la reproduction) est assujettie à sa politique d'utilisation que vous pouvez consulter en ligne.

https://apropos.erudit.org/fr/usagers/politique-dutilisation/ 


\section{$\checkmark$ recensions}

Bertrand Dumas, Dennis GIRA, dir., Le désir en question. Regards bouddhistes et chrétiens. Actes du colloque interreligieux du 5 au 8 juillet 2012 au centre théologique de MeylanGrenoble. Lyon, PROFAC (coll. «PROFAC-Théo », 124), 2015, 186 p.

S'il est un thème délicat, pouvant prêter à interprétations divergentes entre chrétiens et bouddhistes, c'est bien celui du désir. Avant de finalement l'aborder en 2012, le centre théologique de MeylanGrenoble (CTM) en était à sa sixième rencontre entre chrétiens et bouddhistes, et sur des thèmes aussi variés que la non-dualité (1999), l'éthique (2001), la paix (2003), l'hospitalité (2006), le mal et la souffrance (2009). D'autres dialogues similaires s'étaient tenus à l'Institut Karma Ling (Arvillard, Savoie) depuis 1983 sur les thèmes suivants : "Méditation chrétienne et méditation bouddhiste », "Parole et Silence », "Amour et Connaissance », "La spiritualité dans la vie quotidienne », « Dieu et Vacuité », « Trinité et Trikāya ». Un livre comme celui-ci ne s'improvise donc pas : il repose sur une expérience éprouvée de dialogue et provient de personnes de part et d'autre profondément attachées à leurs traditions, habituées à confronter leurs idées et à avancer avec des partenaires « sur un chemin parfois difficile mais tellement enrichissant» (p. 6).

La simple liste des contributions permet d'entrevoir la dynamique d'un livre tout en nuances. Après une introduction par Dennis Gira, «Le désir en question : regards croisés bouddhistes et chrétiens » (p. 5-15), suivent les neuf textes suivants : «Le Christ, un homme de désir» (Dominique Charles, p. 17-31), «Le désir dans la tradition chrétienne» (Bertrand Dumas, p. 33-49), «Le désir en question dans les voies bouddhiques » (Philippe Cornu, p. 51-72), «Regards croisés : une soif nommée désir » (Françoise Bonardel, p. 73-90), "Regards croisés : une perspective chrétienne sur le message bouddhique " (Jacques Scheuer, p. 91-108), "La pratique bouddhique face à la question du désir» (Jigmé Thrinlé Gyatso, p. 109-127), «Les exercices spirituels au risque du désir » (Léo Scherer, p. 128-144), «Les paradoxes du désir» (Paul Magnin, p. 145-162), «Bouddhistes et chrétiens dans leur "être-dialogue" » (Thierry-Marie Courau, p. 163-180).

Il me semble inutile de tenter de résumer ces textes qui souvent se chevauchent ou se complètent, malgré des points de départ différents. Quelques remarques suffiront pour souligner la fécondité de ces réflexions. Tout d'abord, le lecteur se rendra rapidement compte que le désir n'est pas un thème que l'on peut facilement isoler et traiter de façon indépendante. En tant que tel, il mobilise l'ensemble du bouddhisme et l'ensemble du christianisme, quoique de façon différente. Dès lors, on comprendra que ces discussions citent ou évoquent les meilleures ressources de l'une et l'autre tradition, mais parfois trop rapidement pour que le lien entre ces diverses références n'apparaisse toujours nettement. Il ne s'agit évidemment que d'un petit livre qui procède par coups de sonde successifs plutôt qu'une analyse systématique de la question. Mais on déplorera par exemple que la place du désir dans le bouddhisme ancien (en particulier dans le Theravāda) n'ait été qu'effleurée.

La réflexion à propos du désir repose sur une opposition fondamentale, exprimée de façons qui varient selon les écoles de pensée et les traditions. Il y a la soif ou le désir avide facteur d'appropriation, et le désir d'Éveil (cf. p. 54, 62) ; l'attachement qui provient de l'ignorance qui nous fait hypostasier le « je » ou le « moi », et le désir qui sous-tend la vie (p. 111); un désir-passion (qui est 
émotion perturbatrice, un désir fondé sur l'ignorance), et un désir-aspiration (spirituel et altruiste, fondé sur la compréhension) (p. 112); des désirs partiels et multiples, et le grand désir (p. 108, 114) ou le désir plus essentiel (p. 104); le «désir obsessionnel de maîtrise des choses, de soi et des autres ", et la réorientation de "l'énergie psychique fondamentale inhérente à tout homme pour qu'elle devienne désir d'Éveil, désir de liberté, désir de Dieu » (p. 148). Ces multiples expressions, où l'on reconnaît tantôt l'empreinte du bouddhisme tantôt celle du christianisme, montrent qu'il est vain de penser que le but du bouddhisme serait seulement de supprimer le désir alors que le christianisme découvrirait en Dieu comme la quintessence des désirs humains. Le but de la pratique bouddhique est de réduire l'attachement à l'ego bien-aimé (p. 112), mais reste en définitive un élan vers l'ultime liberté.

Même s'ils se retrouvent en même temps à critiquer les désirs humains et à valoriser un désir plus fondamental, les participants à ce colloque me semblent d'accord pour penser que les chemins du bouddhiste et du chrétien diffèrent profondément (cf. p. 148). Scheuer parle plus précisément de «convergence essentielle sur le plan des attitudes spirituelles (non-attachement, désappropriation...), même si les interprétations et les justifications théoriques ou doctrinales sont, le plus souvent, assez différentes et peut-être même peu compatibles » (p. 98). Mais, alors qu'une certaine psychologie emprunte volontiers au bouddhisme des techniques, entre autres de méditation, pour pacifier le moi et le rééquilibrer, on notera la remarque précise du moine tibétain Jigmé Thrinlé Gyatso qui dénonce cette source possible de confusion: "Aussi l'amalgame qui est souvent fait de nos jours entre bouddhisme d'un côté, thérapie et développement personnel de l'autre, est-il incorrect. Ces deux dernières disciplines n'ont pas du tout la même visée : elles proposent de construire et d'équilibrer le sujet, le "moi", pour un mieux-vivre intérieur et extérieur, alors que la voie bouddhiste mène à la réalisation du non-soi » (p. 112). La voie bouddhique apparaît finalement plus rapprochée de la voie chrétienne qu'elle ne l'est d'une psychologie qui tend à sacraliser l'ego et refuse toute forme de dépassement du sujet.

André COUTURE

Université Laval, Québec

Vasundhara Filliozat, La mythologie hindoue, t. I, Viṣnu. Paris, Éditions Āgamāt, 2014, 285 p., avec illustrations ; et t. II, Śiva. Paris, Éditions Āgamāt, 2016, 283 p., avec illustrations.

Comme leur titre général l'indique, ces deux petits livres traitent de la mythologie hindoue. Il ne s'agit pas de livres d'érudition. Pas question d'éditions critiques ou d'annotations savantes, mais plutôt de la vie concrète d'une Inde traditionnelle qui se plaît à écouter des histoires de dieux et de saints, à les raconter, à les chanter, à les peindre et à les sculpter (cf. la préface de P.-S. Filliozat au tome I, p. 9). Ces histoires sont parfois rassemblées en de vastes recueils (épopées, purānas, etc.) où les conteurs puisent inlassablement, comme le fait ici Vasundhara Filliozat, tout en privilégiant le témoignage des villages du Karnātaka dont elle est originaire.

S'il prend ces livres au sérieux, le lecteur attentif découvrira qu'il ne faut pas chercher «la» version authentique d'une histoire de mythologie. Le mythe apparait comme une réalité vivante qui n'a cessé de circuler de par toute l'Inde, et par conséquent de se transformer. Lévi-Strauss en a d'ailleurs fait un principe d'analyse : le mythe se compose de l'ensemble de ses variantes ; il n'y a pas de version authentique ou primitive. Le mythe est bon à penser en chacune de ses versions, car chacune permet d'entrer dans la culture de ceux et celles qui l'acceptent comme tel (cf. Anthropologie structurale, Paris, Plon, 1958, p. 240). Une autre caractéristique de ces livres consiste à mêler inextricablement histoires et images, et indirectement théâtre, musique, rites. C'est que ces 